\title{
Robust Time-Of-Arrival Estimation In Multipath Channels With OFDM Signals
}

\author{
Oded Bialer, Dan Raphaeli, Anthony J. Weiss
}

\begin{abstract}
Time-of-arrival (TOA) estimation of OFDM signals in multipath channels is challenging, especially when the signal bandwidth is limited, such as is the case of WIFI. In this paper, a novel low complexity TOA estimation algorithm is developed for OFDM signals that is robust to various multipath environments. We approximate the received signal as Gaussian with a proposed autocorrelation model, and then derive the Maximum Likelihood estimator with an efficient implementation algorithm. The estimator performance was tested with off-theshelf WIFI 802.11g routers. The results show that the proposed TOA estimator outperforms other known reference methods over various indoor environments.
\end{abstract}

\section{INTRODUCTION}

Orthogonal frequency division multiplexing (OFDM) communications systems are widely deployed, and there is an increase need from these systems to provide position estimation services. Time-of-flight based positioning techniques rely on measuring the signal propagation time between two wireless devices, which essentially requires estimating the received signal time-of-arrival (TOA). In a free space environment, there is a single path between the transmitter and the receiver, and thus estimating the TOA is relatively simple. However, in indoor environment, the transmitted signal is reflected by the walls, floor, ceiling, and other objects. Therefore, there are many dense received replicas of the transmitted signal, which make the TOA estimation of the first signal (that corresponds to the direct line-of-sight path) very challenging, especially in low signal-to-noise (SNR) conditions.

The exact Maximum Likelihood (ML) estimator requires knowing the exact distribution of the multipath. In practice, the multipath first order statistics parameters are unknown, and may vary from one environment to the other. In the Generalized ML (GML) approach, all the multipath coefficients and their arrival times are jointly estimated [4]. In a typical indoor environment, the multipath is dense, the number of paths is large and unknown, and the GML is required to estimate too many parameters. An iterative approximation to the GML was introduced by [5] and referred to in this paper as SCC. In SCC the multipath coefficients are estimated one by one and removed from the received signal sequentially until the strongest remaining coefficient is below a threshold, and the TOA is determined according to the earliest arrival path. However, the successive cancelation is imperfect and, therefore, the error cannot decrease below a certain limit, even as the SNR increases. Other TOA estimation methods that approximate the ML have been developed, but they are not applicable to OFDM signals [6]. A different approach is the "frequency-domain super-resolution TOA estimation", which includes multiple signal classification (MUSIC) [7], and estimation of signal parameters via rotational invariance techniques (ESPRIT) [8]. These subspace methods rely on estimation of the received signal autocorrelation, which requires a large number of statistically independent channel realizations that are not available in many cases. In the absence of statistically independent realizations, spatial smoothing techniques can be applied at the expense of reducing the resolution. Simple approaches for TOA estimation are based on filtering the received signal with a match filter, and then searching for the first time that the match filter energy exceeds a threshold [9]-[12]. These methods have low complexity and perform well when the amplitude of the first arrival component is significantly stronger than the other multipath components, but attain inaccurate TOA estimation when this is not the case, as is often in indoor environments.

The main contribution of this paper is the development of a low complexity TOA estimation algorithm for OFDM signals that is robust to various multipath environments and outperforms other known reference methods in real-life indoor environments.

\section{SYSTEM MODEL}

A standard OFDM scheme is considered in this paper, as depicted in Fig. 1. The OFDM transmitter is presented in Fig. 1(a). A preamble symbol consisting of $L$ frequency bins, denoted by $\boldsymbol{b}$, is transmitted, and it is assumed that $\boldsymbol{b}$ is known to the receiver. An inverse Fourier transform (IFFT) of length $N$ with zero padding of $N-L$ is applied to $\boldsymbol{b}$, which yields the vector $s$ with length $N$. Then a cyclic prefix (CP) of the $K$ last samples in $s$ is added to the beginning of $s$. The signals $x(t)$, $h(t), \eta(t)$ and $r(t)$, in Fig. 1(a), denote the transmitted analog signal, the multipath channel, the white Gaussian additive noise, and the received analog signal, respectively.

The OFDM receiver is shown in Fig. 1(b). The received signal, $r(t)$, is sampled at rate $1 / T_{s}$. A coarse estimation of the preamble TOA in the received samples, $r_{n}$, is applied, and an observation window of $N$ samples, denoted by $\boldsymbol{z}$, is extracted, which is initiated at a time that is within the $\mathrm{CP}$ interval. A fast Fourier transform (FFT) of length $N$ is applied to $\boldsymbol{z}$, which results in $\boldsymbol{y}$ with length $N$, and the preamble TOA offset in the observation window is estimated from $\boldsymbol{y}$.

Fig. 2 illustrates a timing diagram of the observation window $z$ with respect to the $\mathrm{CP}$ and the preamble received signals. The notation $T_{D}$, in the figure, is the maximal channel delay spread, and the notation $\tau$ is the preamble TOA offset in $z$. It is assumed that the CP duration is larger than the sum 
of the channel delay spread and the coarse TOA estimation inaccuracy.

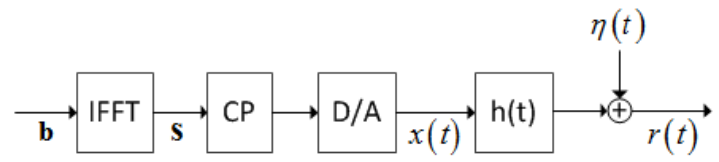

(a)

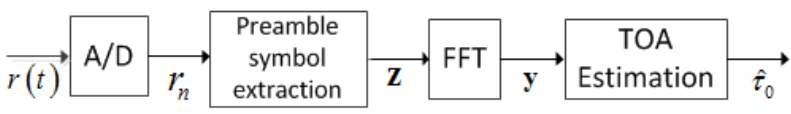

(b)

Fig. 1. Base band system model

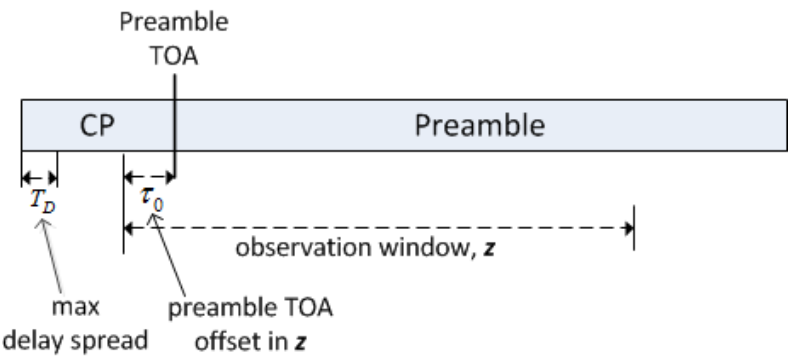

Fig. 2. Preamble observation window, $\boldsymbol{z}$

The channel is assumed to be a time invariant multipath channel with impulse response given by

$$
h(t)=\sum_{m=0}^{M-1} \alpha_{m} \delta\left(t-\tau_{m}\right),
$$

where $M$ is the number of unknown multipath components, $\delta(t)$ is the Dirac delta, $\alpha_{m}$ and $\tau_{m}$ are the random complex coefficient and the random delay of the $m$-th multipath component, respectively. The multipath components are assumed statistically independent.

Without loss of generality, it is assumed that the observation window is initiated at time $t=0$. The preamble TOA within the observation window, $\tau_{0}$, is estimated from the measurement vector, $\boldsymbol{y}$, which can be expressed as

$$
\boldsymbol{y}=\boldsymbol{F} \boldsymbol{z}=\boldsymbol{F} \boldsymbol{q}+\boldsymbol{v}
$$

where

$$
\boldsymbol{F}=\frac{1}{N}\left[\begin{array}{cccc}
1 & 1 & \ldots & 1 \\
1 & e^{j 2 \pi / N} & \ldots & e^{j 2 \pi(N-1) / N} \\
: & : & : & : \\
1 & e^{j 2 \pi(N-1) / N} & \ldots & e^{j 2 \pi(N-1)^{2} / N}
\end{array}\right],
$$

is the discrete Fourier transform (DFT) matrix, $\boldsymbol{q}$ is the noiseless received vector (the signal after passing the multipath channel without noise), and $\boldsymbol{v}$ is the a vector of statistically independent Gaussian noise samples with variance $\sigma_{v}^{2}$.
As for notations, $f(x)$ represents the probability density function of $x$. The superscripts $*, T$ and $H$ represent conjugate, transpose and conjugate transpose, respectively. The notation $\operatorname{diag}\{\boldsymbol{x}\}$ represents a diagonal matrix whose diagonal entries are the elements in vector $\boldsymbol{x}$. The notation $\boldsymbol{I}$ is used for the identity matrix.

\section{TOA Estimation ALgORITHM}

Next, we derive an estimator for the preamble TOA, $\tau_{0}$ from the measurement vector $\boldsymbol{y}$. The noiseless received vector of samples in the time domain, $\boldsymbol{q}$, can be expressed as

$$
\boldsymbol{q}=\sum_{m=0}^{M-1} \alpha_{m} \boldsymbol{\psi}\left(\boldsymbol{s}, \tau_{m}\right)
$$

where $\psi\left(s, \tau_{m}\right)$ is a fractional cyclic shift rotation of vector $s$ by time $\tau_{m}$, which can be expressed by

$$
\boldsymbol{\psi}\left(\boldsymbol{s}, \tau_{m}\right)=\boldsymbol{F}^{H} \boldsymbol{G}_{\tau_{m}} \boldsymbol{F} \boldsymbol{s},
$$

where

$$
\boldsymbol{G}_{\tau}=\operatorname{diag}\left\{1, e^{j 2 \pi \tau / N}, e^{j 2 \pi 2 \tau / N}, . ., e^{j 2 \pi(N-1) \tau / N}\right\}
$$

is a linear phase shift operator.

By substituting (4) and (5) into (2) and making use of the fact that $\boldsymbol{F} \boldsymbol{F}^{H}=\boldsymbol{I}$, we have that

$$
\boldsymbol{y}=\sum_{m=0}^{M-1} \alpha_{m} \boldsymbol{\xi}_{\tau_{m}}+\boldsymbol{v}
$$

where

$$
\boldsymbol{\xi}_{\tau_{m}}=\boldsymbol{G}_{\tau_{m}} \boldsymbol{F} \boldsymbol{s}
$$

Since $\alpha_{m}$ are statistically independent, then from (7) it is realized that $\boldsymbol{y}$ is equal to a sum of statistically independent vectors. The central limit theorem (CLT) for random vectors [13] states that the sum of independent random vectors is a Gaussian vector when the number of the summed vectors approaches infinity. Typically, in indoor environments there are many dense arrivals. Therefore, we make use of the CLT and approximate $\boldsymbol{y}$ as Gaussian, and thus

$$
f\left(\boldsymbol{y} \mid \tau_{0}\right)=\frac{1}{\pi^{N}\left|\boldsymbol{R}\left(\tau_{0}\right)\right|} e^{-\boldsymbol{y}^{H} \boldsymbol{R}^{-1}\left(\tau_{0}\right) \boldsymbol{y}},
$$

where

$$
\boldsymbol{R}\left(\tau_{0}\right)=\mathrm{E}\left\{\boldsymbol{y} \boldsymbol{y}^{H} \mid \tau_{0}\right\} .
$$

The Maximum Likelihood (ML) TOA estimator is given by

$$
\hat{\tau}_{0}=\arg \max _{\tau_{0}} f\left(\boldsymbol{y} \mid \tau_{0}\right) .
$$

By substituting (9) into (11), applying $\ln$ and multiplying by -1 , we can express the TOA ML estimator as

$$
\hat{\tau}_{0}=\arg \min _{\tau_{0}}\left\{\ln \left(\left|\boldsymbol{R}\left(\tau_{0}\right)\right|\right)+\boldsymbol{y}^{H} \boldsymbol{R}^{-1}\left(\tau_{0}\right) \boldsymbol{y}\right\} .
$$

Calculating the estimator in (12) requires to know $\boldsymbol{R}\left(\tau_{0}\right)$, for each $\tau_{0}$. The complexity of calculating the cost function in (12), for each TOA hypothesis, is $O\left(N^{3}\right)$ (determined by the complexity of evaluating $R^{-1}\left(\tau_{0}\right)$ ), and for a grid search 
resolution that is larger by a factor $P$ than the sampling rate, there are all together $P \times N$ TOA hypotheses, and therefore the complexity of the estimator in (12) is $O\left(P N^{4}\right) \approx O\left(N^{4}\right)$, assuming $P \ll N$.

Next, we will continue to derive (12) in order to obtain an analytical expression for $\boldsymbol{R}\left(\tau_{0}\right)$, and also to obtain an efficient implementation of the estimator with lower complexity. By making use of the relation $\boldsymbol{G}_{\tau_{m}}=\boldsymbol{G}_{\tau_{0}} \boldsymbol{G}_{\tau_{m}-\tau_{0}}$, we can further express (7) as

$$
\boldsymbol{y}=\boldsymbol{G}_{\tau_{0}} \sum_{m=0}^{M-1} \alpha_{m} \boldsymbol{G}_{\tau_{m}-\tau_{0}} \boldsymbol{F} \boldsymbol{s}+\boldsymbol{v}=\boldsymbol{G}_{\tau_{0}} \boldsymbol{F} \boldsymbol{q}_{0}+\boldsymbol{v},
$$

where

$$
\boldsymbol{q}_{0}=\sum_{m=0}^{M-1} \alpha_{m} \boldsymbol{F}^{H} \boldsymbol{G}_{\tau_{m}-\tau_{0}} \boldsymbol{F} \boldsymbol{s}=\sum_{m=0}^{M-1} \alpha_{m} \boldsymbol{\psi}\left(\boldsymbol{s}, \tau_{m}-\tau_{0}\right),
$$

is the observation window of the noiseless received samples when the preamble TOA is perfectly aligned with the beginning of the observation window. By substituting (13) into (10), and making use of the relation

$$
\boldsymbol{G}_{\tau_{0}} \boldsymbol{G}_{\tau_{0}}^{H}=\boldsymbol{G}_{\tau_{0}}^{H} \boldsymbol{G}_{\tau_{0}}=\boldsymbol{I},
$$

we have

$$
\boldsymbol{R}\left(\tau_{0}\right)=\boldsymbol{G}_{\tau_{0}}\left(\boldsymbol{F} \boldsymbol{R}_{0} \boldsymbol{F}^{H}+\sigma_{v}^{2} \boldsymbol{I}\right) \boldsymbol{G}_{\tau_{0}}^{H},
$$

where

$$
\boldsymbol{R}_{0}=\mathrm{E}\left\{\boldsymbol{q}_{0} \boldsymbol{q}_{0}^{H}\right\} .
$$

From (16) and (15), we get

$$
\begin{array}{r}
\ln \left(\left|\boldsymbol{R}\left(\tau_{0}\right)\right|\right)=\ln \left(\left|\boldsymbol{G}_{\tau_{0}}^{H} \boldsymbol{G}_{\tau_{0}}\left(\boldsymbol{F} \boldsymbol{R}_{0} \boldsymbol{F}^{H}+\sigma_{v}^{2} \boldsymbol{I}\right)\right|\right)= \\
\ln \left(\left|\boldsymbol{F} \boldsymbol{R}_{0} \boldsymbol{F}^{H}+\sigma_{v}^{2} \boldsymbol{I}\right|\right),
\end{array}
$$

which is independent of the TOA hypothesis $\tau_{0}$, and hence it can be eliminated from the TOA metric in (12). By substituting (16) into (12), we have

$$
\hat{\tau}_{0}=\arg \min _{\tau_{0}}\left\{\boldsymbol{y}^{H} \boldsymbol{G}_{\tau_{0}} \boldsymbol{D} \boldsymbol{G}_{\tau_{0}}^{H} \boldsymbol{y}\right\}
$$

where

$$
\boldsymbol{D}=\left(\boldsymbol{F} \boldsymbol{R}_{0} \boldsymbol{F}^{H}+\sigma_{v}^{2} \boldsymbol{I}\right)^{-1} .
$$

Assuming that $\boldsymbol{D}$, which is independent of $\tau_{0}$, is calculated in advanced and stored in memory, then the complexity of calculating the cost function in (19) for each TOA hypothesis is $O\left(N^{2}\right)$. Assuming that there are $P \times N$ TOA hypotheses, then the complexity of the estimator is $O\left(P N^{3}\right)$. Next, we derive an efficient implementation of the estimator with reduced complexity.

\section{A. Efficient Implementation}

We now further derive (19) in order to obtain an efficient implementation of the estimator. We make use of the relation

$$
\boldsymbol{y}^{H} \boldsymbol{G}_{\tau_{0}}=\boldsymbol{g}_{\tau_{0}}^{H} \boldsymbol{Y}^{H}
$$

where

$$
\boldsymbol{Y}=\operatorname{diag}\{\boldsymbol{y}\}
$$

$$
\boldsymbol{g}_{\tau_{0}}=\left[\begin{array}{lllll}
1 & \omega & \omega^{2} & \ldots & \omega^{N-1}
\end{array}\right]^{T},
$$

and $\omega=e^{-j 2 \pi \tau_{0} / N}$. By substituting (21) into (19), we have

$$
\hat{\tau}_{0}=\arg \min _{\tau_{0}} \boldsymbol{g}_{\tau_{0}}^{H} \boldsymbol{C g}_{\tau_{0}}
$$

where

$$
\boldsymbol{C}=\boldsymbol{Y}^{H} \boldsymbol{D} \boldsymbol{Y}
$$

It can be shown that

$$
\boldsymbol{g}_{\tau_{0}}^{H} \boldsymbol{C g}_{\tau_{0}}=2 \text { Real }\left\{0.5 u_{0}+\sum_{i=1}^{N-1} u_{i} \omega^{i}\right\},
$$

where $u_{i}$ is the sum of the $i$-th diagonal of $\boldsymbol{C}$, i.e.,

$$
u_{i}=\sum_{n=0}^{N-1-i} c_{n, n+i} \quad ; \quad i=\{0,1, . ., N-1\},
$$

and $c_{i, j}$ is the element in the $i$-th row and $j$-th column of $\boldsymbol{C}$.

From (24)-(26), the ML TOA estimator can be efficiently implemented by applying IFFT to the vector $\boldsymbol{u}=$ $\left[\begin{array}{llll}0.5 u_{0} & u_{1} & \ldots & u_{N-1}\end{array}\right]^{T}$, and then choosing the TOA that corresponds to the minimal real sample. The TOA metric search grid resolution (search grid of $\tau_{0}$ ) can be conveniently increased by applying zero padding to $\boldsymbol{u}$ prior to the IFFT. The TOA estimation algorithm and its complexity is summarized below.

\section{TOA estimation algorithm}

- Off-line settings:

1) Determine $\boldsymbol{R}_{0}$ (see section III-B).

2) Calculate the matrix $\boldsymbol{D}$ given in (20), and store it in memory.

- TOA estimation for search grid resolution of $T_{s} / P(P$ times smaller spacing than the sampling rate):

1) Calculate the vector $\boldsymbol{u}$ from (25) and (27). The complexity is $O\left(N^{2}\right)$, since $\boldsymbol{Y}$ is diagonal and $\boldsymbol{D}$ is pre-calculated and stored in memory.

2) Apply zero padding of $(P-1) N$ zeros to $\boldsymbol{u}$, calculate IFFT of size $P N$, and keep only the real samples of the result, denoted as $\gamma$. The complexity is $O\left(P N \log _{2}(P N)\right)$.

3) Search for the index of the smallest sample in $\gamma$, denoted as $\rho$, and determine the TOA estimation as $\hat{\tau}_{0}=\rho T_{s} / P$. The complexity is $O(P N)$.

The total algorithm complexity is $O\left(N^{2}+P N \log _{2}(P N)+\right.$ $P N)$. Typically $P \ll N$, and in this case, the overall algorithm complexity is $O\left(N^{2}\right)$, which is significantly smaller than the complexity of implementing (19), which is $O\left(N^{3}\right)$.

\section{B. Determining $\boldsymbol{R}_{0}$}

The TOA estimator in (19) and (24) requires knowing $\boldsymbol{R}_{0}$. Measuring $\boldsymbol{R}_{0}$ empirically requires to collect the received samples at many different positions per each different environment, and also requires having the correct first arrival time for each measurement. Therefore, obtaining $\boldsymbol{R}_{0}$ empirically is very complex, and thus is impractical. Instead, we derive next, an analytical expression that approximates $\boldsymbol{R}_{0}$.

We consider the multipath arrivals to be confined to a finite time grid, with resolution (grid 
spacing) of $\Delta$, which is sufficiently small. Let $\Psi=\left[\begin{array}{lllll}\psi(s, 0) & \psi(s, \Delta) & \psi(s, 2 \Delta) & \ldots & \psi\left(s, T_{D}\right)\end{array}\right]$, where $T_{D}$ is the maximal delay spread, then from (14) we can express $\boldsymbol{q}_{0}$ by

$$
\boldsymbol{q}_{0}=\boldsymbol{\Psi} \boldsymbol{h}
$$

where $\boldsymbol{h}$ is a vector of channel samples with spacing $\Delta$, starting from the first arrival time, $\tau_{0}=0$, until the maximal delay spread $T_{D}$, i.e., $\boldsymbol{h}=\left[\begin{array}{lllll}h(0) & h(\Delta) & h(2 \Delta) & \ldots & h\left(T_{D}\right)\end{array}\right]$. We note that the sampling rate of $\psi\left(s, \tau_{m}\right)$ is $T_{s}$, which is in practise significantly larger than $\Delta$, and thus in this case the number of columns in $\boldsymbol{\Psi}$ is significantly larger than the number of its rows.

By substituting (28) into (17), we obtain

$$
\boldsymbol{R}_{0}=\boldsymbol{\Psi} \Lambda \Psi^{H}
$$

where $\boldsymbol{\Lambda}=E\left\{\boldsymbol{h} \boldsymbol{h}^{H}\right\}$. The channel samples are assumed statistically independent, therefore we have

$$
\boldsymbol{\Lambda}=\operatorname{diag}\left\{\sigma_{h}^{2}(0,0), \sigma_{h}^{2}(\Delta, 0), \sigma_{h}^{2}(2 \Delta, 0), . ., \sigma_{h}^{2}\left(T_{D}, 0\right)\right\},
$$

where $\sigma_{h}^{2}\left(t, \tau_{0}\right)=E\left\{|h(t)|^{2} \mid \tau_{0}\right\}$ is the channel power delay profile.

In (29), we have obtained an expression for $\boldsymbol{R}_{0}$ that is a function of the known transmitted signal $s$, and the channel power delay profile $\sigma_{h}^{2}\left(t, \tau_{0}\right)$. In common multipath channel models [1]-[3], the multipath channel is composed of clusters, where the energy of the components in the cluster decays exponentially with the delay, and also the energy of the entire cluster decays exponentially with the cluster delay. In typical indoor environments, the clusters arrivals are dense, and therefore we approximate the power delay profile by

$$
\sigma_{h}^{2}\left(t, \tau_{0}\right) \approx \mu_{0} \delta\left(t-\tau_{0}\right)+e^{-\left(t-\tau_{0}\right) / \mu_{1}} u\left(t-\tau_{0}\right)
$$

where $\mu_{0} \geq 0, \mu_{1} \geq 0$, and $u(t)$ denotes a step function given by

$$
u(t)=\left\{\begin{array}{ll}
0 & t \leq 0 \\
1 & t>0
\end{array}\right\} .
$$

The simplified model in (31) includes a LOS component at time $t=\tau_{0}$, and an exponential decaying function for $t>\tau_{0}$. We note that other more complicated power delay profile models were also tested and did not attain a significant performance advantage. The parameters, $\mu_{0}, \mu_{1}$, have physical interpretation that depend upon the characteristics of the environment, as explained in the following, and therefore can be predicted, to some extent, without measuring the channel power delay profile empirically for each environment.

The parameter $\mu_{1}$ is related to the channel delay spread, which is a function of the environment area size. On average, $\mu_{1}$ increases as the area of the indoor environment increases, since there are reflections with longer delay. The parameter $\mu_{0}$, reflects the strength of the direct LOS path with respect to the following arrival paths. The energy of the first arrival component is proportional to the distance between the transmitter and the receiver, while the average energy from all the reflecting objects is approximately constant and independent of the distance. Under this approximation, $\mu_{0}$ is inversely proportional to the average distance between the transmitter and the receiver in the environment.

\section{RESUlTS AND Discussion}

Next, we present the TOA estimation performance obtained with a test system, for two different indoor multipath environments. The test system had a universal software radio peripheral device [15] that recorded transmissions from offthe-shelf 802.11g WIFI routers [14]. The TOA of the received preamble was estimated in various locations and compared to the true TOA ${ }^{1}$. In each environment, the TOA estimation algorithm detailed in Sections III-A - III-B, referred to as the Gaussian Estimator (GE), was tested against three reference methods: MUSIC [7], successive component cancelation SCC [5], and jump back search forward (JBSF) [10]. In the JBSF method, the channel estimation was converted to the time domain and then the TOA was estimated by a search forward, from the beginning of the observation window until the sample with peak energy, for the first sample with energy that exceeds a threshold level. The performance comparison criteria was the TOA estimation error's cumulative distribution function (CDF).

The two tested indoor environments were: office floor, and conference hall, shown in Fig. 3, and Fig. 4, respectively. In these figures, the position of the transmitting WIFI router is marked by star sign, and the receiving unit was located at 100 different positions, approximately spread uniformly in all the gray shaded areas in the figures. Both test environments, had multipath reflections from the walls, floor, ceiling, and furniture. The channel power delay profile parameters, $\mu_{0}$ and $\mu_{1}$ were optimized according to the environment characteristics, as explained in III-B. Meaning, the environment area, and the average distance between transmitter and receiver.

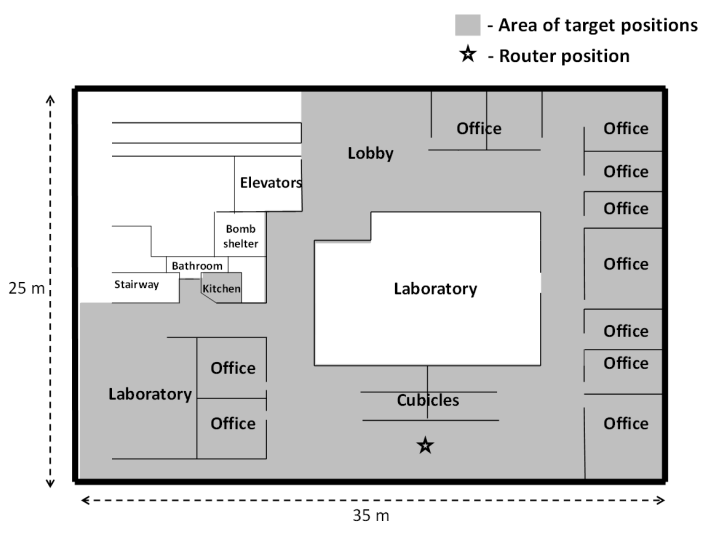

Fig. 3. Diagram of office floor test site

The CDF results are presented in Figures 5, and 6. It is shown that the GE attains smaller error than the reference methods for both test environments and for any given CDF probability. At probability of 0.9 , the GE attains an error

\footnotetext{
${ }^{1}$ the true TOA was measured by a parallel cable link
} 


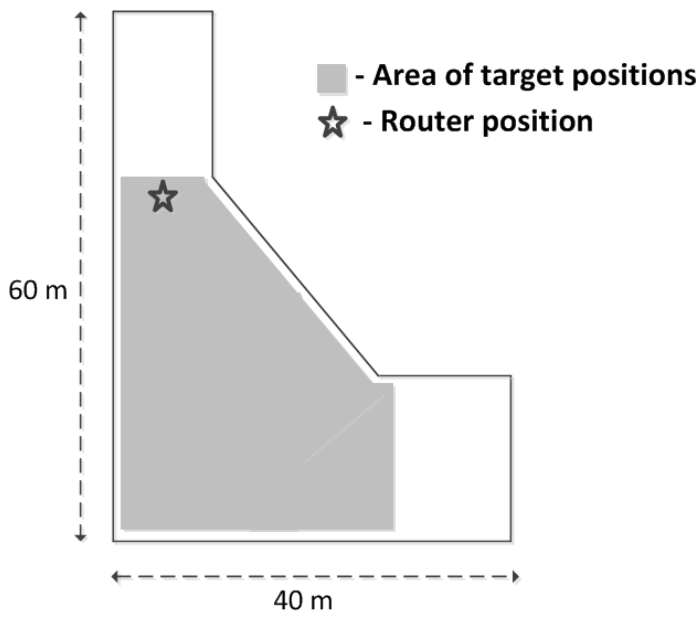

Fig. 4. Diagram of conference hall test site

smaller by a factor between 2 and 3 compared to the MUSIC method, which was the reference method with best performance. It is important to note, that the performance of the proposed estimator compared to the reference methods was tested in other multipath environments, and similar performance advantages as in Figures 5- 6 were observed. These results are not presented here due to limited space.

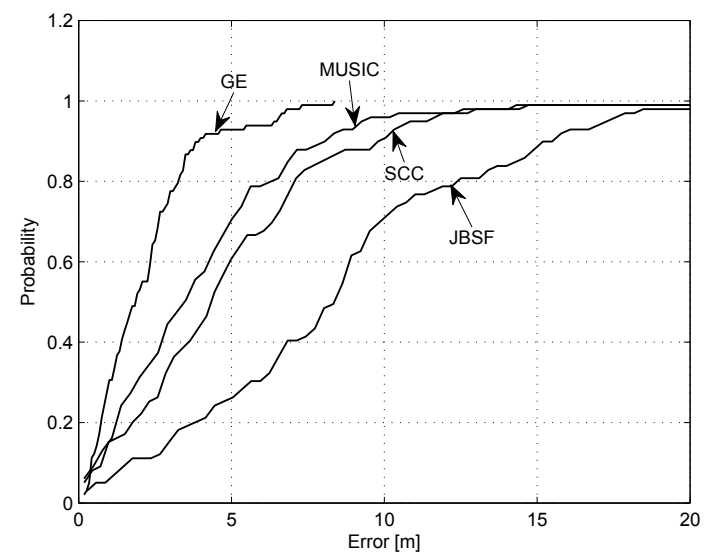

Fig. 5. TOA estimation error CDF for measurements of WIFI $802.11 \mathrm{~g}$ in an office floor environment.

\section{CONCLUSIONS}

A novel TOA estimation algorithm was developed for OFDM signals. The estimator can conveniently be adjusted based on the environment characteristics, such as the area size and the average distance between the transmitter and the receiver, and hence is robust to various multipath environments. Furthermore, the estimation algorithm has low computational complexity and is practical for implementation. The estimator's results showed a significant performance advantage compared to known reference methods in real-life multipath environments. With probability of 0.9 , the novel estimator

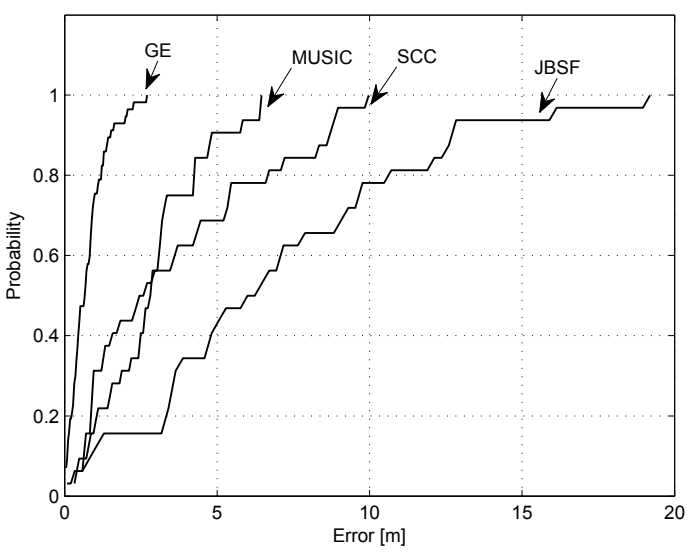

Fig. 6. TOA estimation error CDF for measurements of WIFI $802.11 \mathrm{~g}$ in an large indoor conference hall.

attained an error smaller by more than factor 2 compared to other known reference methods.

\section{REFERENCES}

[1] A. M. Saleh and R. A. Valenzuela, "A Statistical Model for Indoor Multipath Propagation," IEEE J. Selected. Areas Commun., vol. SAC-5, no. 2, pp. 128-137, Feb. 1987.

[2] A. F. Molisch, "Ultrawideband Propagation Channels-Theory, Measurements, and Modeling," IEEE Trans. on Vehicular Technology, vol. 54, no. 5, pp. 1528-1545, Sep. 2005.

[3] A. F. Molisch et al., "A Comprehensive Standardized Model for Ultrawideband Propagation Channels," IEEE Trans. on Antennas and Propagation, vol. 54, no. 11, pp. 3151-3166, Nov. 2006

[4] M. Z. Win and R. A. Scholtz, "Characterization of ultra-wide bandwidth wireless indoor communications channels: A communication theoretic view," IEEE J. Sel. Areas Commun., vol. 20, pp.1613-1627, Dec. 2002.

[5] J. Y. Lee and R. A. Scholtz, "Ranging in a dense multipath environment using an UWB radio link," IEEE J. Select. Areas Commun., vol. 20, no. 9, pp. 1677-1683, Dec. 2002.

[6] O. Bialer, D. Raphaeli, A. J. Weiss, 'Efficient Time of Arrival Estimation Algorithm Achieving Maximum Likelihood Performance in Dense Multipath,", IEEE Trans. Signal Processing, vol 60, no. 3, pp. 1241-1252, March 2012.

[7] X. Li, "Super-Resolution TOA Estimation With Diversity for Indoor Geolocation," IEEE Trans. Wireless Commun., vol. 3, no. 1, Jan. 2004

[8] R. Roy, A. Paulraj and T. Kailath, "Estimation of Signal Parameters via Rotational Invariance Techniques-ESPRIT", IEEE Trans. Acoust., Speech, Signal Process., vol. 37, no. 7, pp. 984-995, Jul. 1989.

[9] L. Stoica, A. Rabbachin, and I. Oppermann, "A low-complexity noncoherent IR-UWB transceiver architecture with TOA estimation," IEEE Trans. Microwave Theory Tech., vol. 54, pp. 1637-1646, Jun. 2006.

[10] I. Guvenc and Z. Sahinoglu, "Threshold-based TOA estimation for impulse radio UWB systems," IEEE int. Conf. Ultra-Wideband (ICUWB), Zurich, Switzerland, 2005, pp. 420-425.

[11] D. Dardari, A. Conti, U. Ferner, A. Giorgetti, M. Z. Win, ’Ranging With Ultrawide Bandwidth Signals in Multipath Environments," IEEE Special Issue on UWB Technology and Emerging Applications, vol. 97, no. 2, pp. 404-426, Feb. 2009.

[12] I. Guvenc and Z. Sahinoglu, "Multiscale Energy Products for TOA Estimation in IR-UWB Systems,' IEEE Global Telecommun. Conf., St. Louis, MO, vol. 1, pp. 209-213, Dec. 2005.

[13] R. C. Mittelhammer, Mathematical Statistics For Economics And Business, Chapter 5.5, Springer-Verlag New York Inc., 1996.

[14] D. Vassis, G. Kormentzas, A. Rouskas, I. Maglogiannis, "The IEEE $802.11 \mathrm{~g}$ standard for high data rate WLANs," IEEE Network, vol.19, no.3, pp.21-26, May-June 2005.

[15] Ettus Research, "USRP N200/N210 Network Series," Avialable online: http://www.ettus.com/content/files/kb/Ettus_Networked_Series.pdf. 